\title{
Effect of 5-aminolevulinic acid on the haem biosynthesis pathway in pancreatic cancer and pancreatic ductal epithelial cell lines
}

Peter L. Labib, Walid Al-Akkad, Brian R. Davidson, Alexander J. MacRobert, Stephen P. Pereira

Peter L. Labib, Walid Al-Akkad, Brian R. Davidson, Alexander J. MacRobert, Stephen P. Pereira, "Effect of 5-aminolevulinic acid on the haem biosynthesis pathway in pancreatic cancer and pancreatic ductal epithelial cell lines," Proc. SPIE 11070, 17th International Photodynamic Association World Congress, 110706K (7 August 2019); doi: 10.1117/12.2525085 


\title{
Effect of 5-aminolevulinic acid on the haem biosynthesis pathway in pancreatic cancer and pancreatic ductal epithelial cell lines
}

\author{
Peter L. Labib*a, Walid Al-Akkad ${ }^{\mathrm{a}}$, Brian R. Davidson ${ }^{\mathrm{b}}$, \\ Alexander J. MacRobert ${ }^{\mathrm{b}}$, Stephen P. Pereira ${ }^{\mathrm{a}}$ \\ ${ }^{a}$ Institute for Liver \& Digestive Health, University College London (Royal Free Campus), Royal

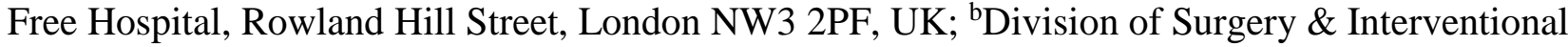 \\ Science, University College London (Royal Free Campus), Royal Free Hospital, Rowland Hill \\ Street, London NW3 2PF, UK
}

\begin{abstract}
Introduction: 5-aminolevulinic acid (ALA) generates protoporphyrin IX (PpIX)-induced fluorescence by acting as a substrate for the haem biosynthesis pathway. Despite suggestions that ALA could be used for pancreatic cancer photodiagnostics, the pancreatic cancer cell line PANC-1 only shows weak fluorescence following ALA administration. A possible explanation was that the haem biosynthesis pathway varies between cancers.

Methods: We compared the mRNA expression of the haem biosynthesis pathway of PANC-1 (weak fluorescence) with the pancreatic cancer cell line CFPAC-1 (strong fluorescence) and the pancreatic ductal cell line H6c7 (control) with or without 24 hours ALA incubation. Cells were seeded on day one, fresh media with or without ALA (0.5mM) added on day two, and RNA extracted on day three. Quantitative real-time polymerase chain reaction was performed to assess the relative mRNA expression of four membrane transporters and eight enzymes responsible for haem biosynthesis.

Results: Post-ALA incubation, CFPAC-1 demonstrated significant downregulation of cell membrane ALA influx transporter PEPT1, downregulation of ALA synthase and upregulation of the mitochondrial membrane transporter ABCB6. PANC-1, whilst showing similar changes to ALA synthase and ABCB6, showed significant upregulation of the PpIX efflux transporter ABCG2. PANC-1 also had minimal PEPT1 expression pre- and post-ALA. H6c7 demonstrated significant up- or downregulation of three transporters and five enzymes.

Conclusion: Poor PpIX-induced fluorescence in PANC-1 is likely to be secondary to decreased ALA influx from low PEPT1 expression and increased ABCG2 expression. The use of nanocarriers to deliver ALA and/or ABCG2 inhibitors may improve ALA-induced fluorescence in PANC-1 and other ALA-resistant cancers.
\end{abstract}

Keywords: 5-aminolevulinic acid, ALA, haem biosynthesis pathway, pancreatic cancer, photodiagnostics, PEPT1, ABCG2

\section{INTRODUCTION}

\section{Pancreatic cancer}

Pancreatic cancer is the $11^{\text {th }}$ most common cancer in the U.K. and the $6^{\text {th }}$ most common cause of cancer death ${ }^{1}$. A combination of aggressive tumor biology and late onset of symptoms means that most patients (60\%) are diagnosed at an advanced stage ${ }^{2}$. Due to the late stage at diagnosis, only $10 \%$ of patients will undergo curative-intent surgery and only $20 \%$ will receive chemotherapy ${ }^{2}$. Whilst complete surgical resection can offer a chance of cure, recurrence is common with palliative treatments only providing a modest survival benefit ${ }^{3,4}$. Strategies to improve survival include earlier diagnosis through non-invasive or invasive diagnostic tests, adjuncts to current surgical techniques that improve intraoperative identification of unresected disease, and the use of locoregional therapies in the palliative setting.

\section{5-aminolevulinic acid}

5-aminolevulinic acid (ALA) is a clinically-approved photosensitiser that could play a role in these strategies. ALA is a

*peter.labib.16@ucl.ac.uk; phone +44 207433 2894; https://www.ucl.ac.uk/liver-and-digestive-health/

17th International Photodynamic Association World Congress, edited by Tayyaba Hasan, Proc. of SPIE Vol. 11070, 110706K - C 2019 SPIE · CCC code: 0277-786X/19/\$21 - doi: 10.1117/12.2525085 
naturally occurring amino acid that forms an early substrate in the haem biosynthesis pathway (Figure 1). Protoporphyrin IX (PpIX), the penultimate molecule in the pathway, fluoresces red when excited with blue/violet light with a peak emission at $635 \mathrm{~nm}^{5}$. The addition of exogenous ALA to the haem synthesis pathway leads to an accumulation of PpIX that can be exploited for the purpose of photodynamic diagnostics (PDD) and photodynamic therapy (PDT). Although not specifically targeted, there is preferential accumulation of ALA-induced PpIX fluorescence in neoplastic tissues when compared to surrounding non-neoplastic tissues ${ }^{6,7}$. The reasons for this are complex and include morphological, cell-cycle, enzymatic and environmental factors ${ }^{6}$. Enzymatic examples observed in some cancers include increased porphobilinogen deaminase activity increasing the conversion of porphobilinogen to hydroxymethylbilane, and reduced ferrochelatase activity leading to PpIX accumulation ${ }^{6}$.

Figure 1 The haem biosynthesis pathway

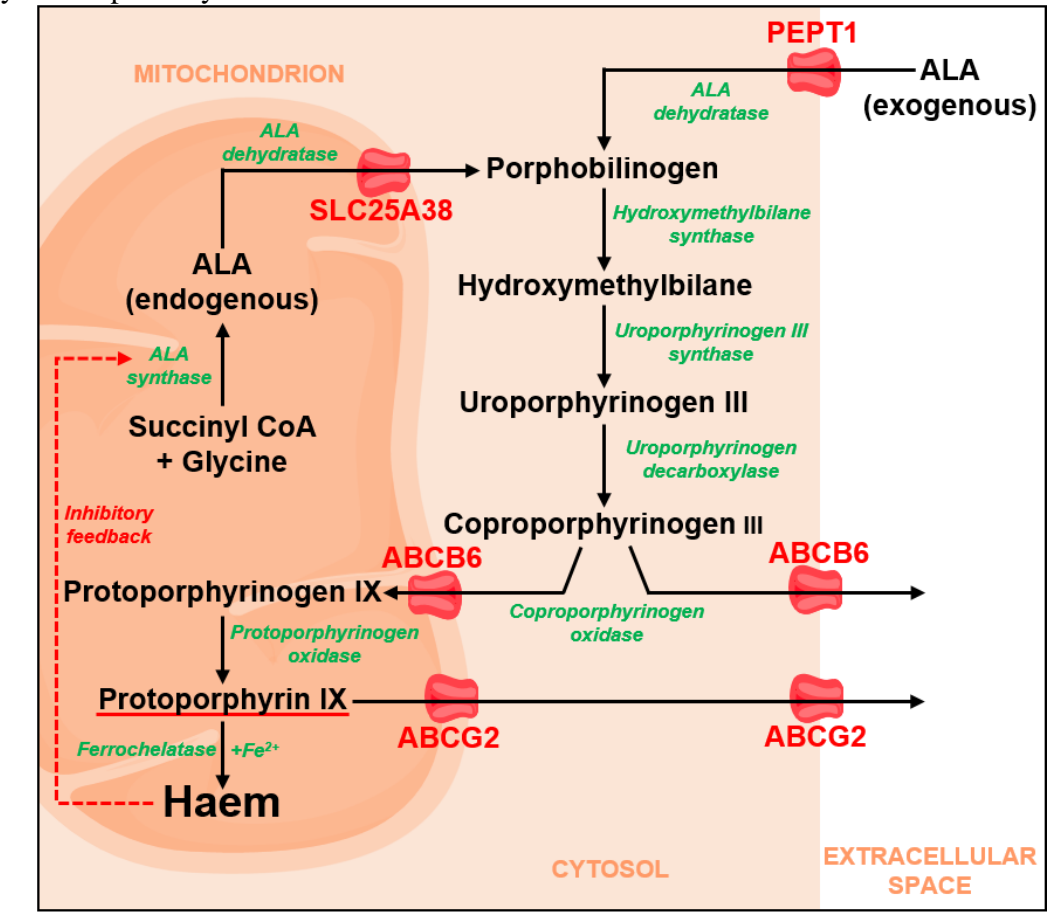

Image constructed using icons from Servier Medical Art (licensed under CC BY 3.0)

\begin{abstract}
Aims
Whilst researching the fluorescence effect of ALA on pancreatic cancer cell lines, it was noted that whilst the pancreatic cancer cell line CFPAC-1 readily converted ALA into PpIX, the pancreatic cancer cell line PANC-1 did not. Our aim was to elucidate the relative mRNA expression of the transporters and enzymes involved in the haem biosynthesis pathway in these cell lines, with or without 24 hours dark incubation with ALA. The immortalized non-tumorigenic pancreatic ductal cell line H6c7, that also shows low ALA-induced PpIX fluorescence, was used as a non-neoplastic control.
\end{abstract}

\title{
2. MATERIALS AND METHODS
}

\section{Cell culture}

PANC-1 was purchased from RIKEN BioResource Centre (RIKEN BRC, Tsukuba, Japan), CFPAC-1 from ECACC (European Collection of Authenticated Cell Cultures, Salisbury, United Kingdom) and H6c7 from Kerafast (Kerafast, Inc., Boston, USA). PANC-1 was cultured in Dulbecco's modified Eagle's medium containing $4.5 \mathrm{~g} \mathrm{~L}^{-1} \mathrm{glucose}^{-1} \mathrm{and}_{0.58} \mathrm{~g} \mathrm{~L}^{-1}$ L-glutamine (Lonza $\left.{ }^{\circledR}\right)$ supplemented with $10 \%$ Fetal Bovine Serum (FBS) (Gibco ${ }^{\mathrm{TM}}$ ). CFPAC-1 was cultured in Iscove's modified Dulbecco's medium containing $4.5 \mathrm{~g} \mathrm{~L}^{-1}$ glucose, $4.0 \mathrm{mM}$ L-glutamine and HEPES buffer (Gibco ${ }^{\mathrm{TM}}$ ) supplemented with $10 \%$ FBS. H6c7 was cultured in Keratinocyte serum free media with L-glutamine, epidermal growth factor, bovine pituitary extract $\left(\mathrm{Gibco}^{\mathrm{TM}}\right)$ and $1 \%$ antibiotic/antimycotic. Cells were cultured in $75 \mathrm{~cm}^{2}$ tissue culture flasks (Techno Plastic Products $®$ ) in a humidified incubator at $37.0^{\circ} \mathrm{C}$ with $5 \% \mathrm{CO}_{2}$. Cells were trypsinized when $\sim 80 \%$ confluent with $0.5 \%$ trypsin-EDTA $\left(\mathrm{Gibco}^{\mathrm{TM}}\right)$ and passaged at a split ratio of 1:4. 


\section{Quantitative Polymerase Chain Reaction (qPCR)}

Each cell line had two conditions: control (serum-free media for 24 hours) or ALA (0.5mM ALA in serum-free media for 24 hours). Cells were seeded on $10 \mathrm{~cm}^{2}$ petri dishes (one million cells per dish) on day 0 . On day 1 the culture medium was removed and replaced with serum-free medium with or without $0.5 \mathrm{mM}$ ALA and incubated in conditions preventing light exposure. After 24 hours, the cells were washed with Dulbecco's Phosphate Buffered Saline (PBS), removed using a cell scraper and transferred to $15 \mathrm{ml}$ centrifuge tubes. RNA extraction protocol (RNeasy minikit, QIAGEN) was as follows: After centrifugation, the supernatant was discarded and cells resuspended in $600 \mu 1$ of buffer RLT/B-mercaptoethanol. $700 \mu 1$ ethanol was added to the cell suspension and samples spun in minispin columns to discard the supernatant. This step

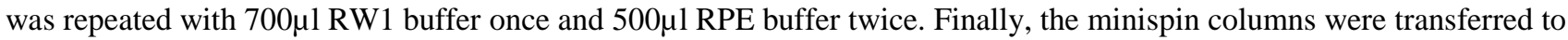
$1.5 \mathrm{ml}$ microfuge tubes with $50 \mu \mathrm{l}$ RNase-free water and centrifuged to extract RNA. RNA was quantified using spectrophotometry (NanoDrop ${ }^{\mathrm{TM}}$ 2000). Complementary DNA (cDNA) was synthesized using High Capacity cDNA Reverse Transcription (RT) Kit (Applied Biosystems®). RNA samples were diluted with RNA-free water to a concentration of $100 \mathrm{ng} / \mu \mathrm{l}$, combined with Mastermix prepared to manufacturer instructions and reverse transcription performed using a Q-Cycler II thermal cycler (Quanta Biotech Ltd.). qPCR was performed using the ABI 7500 Fast Real time PCR System (Applied Biosystems ${ }^{\circledR}$ ) using custom TaqMan array plates. The probes targeted the cDNA of the following mRNA sequences:

- Enzymes: ALA synthase, ALA dehydratase, hydroxymethylbilane synthase, uroporphyrinogen III synthase, uroporphyrinogen III decarboxylase, coproporphyrinogen oxidase, protoporphyrinogen oxidase, ferrochelatase.

- Transporters: PEPT1, ABCB6, ABCG2, SLC25A38.

Relative mRNA expression was normalized to the housekeeping gene HPRT1.

\section{RESULTS}

Changes in relative mRNA expression for the three cell lines are presented in Figure 2. Post-ALA incubation, CFPAC-1 demonstrated significant downregulation of cell membrane ALA influx transporter PEPT1, downregulation of ALA synthase and upregulation of the mitochondrial and cell membrane transporter ABCB6. PANC-1, whilst showing similar changes to ALA synthase and ABCB6, also showed significant upregulation of the PpIX efflux transporter ABCG2. PANC-1 had minimal PEPT1 expression in either experimental condition. H6c7 also demonstrated significant downregulation of ALA synthase, as well as significant upregulation of three transporters (SCL25A38, ABCB6 and ABCG2) and four enzymes (uroporphyrinogen decarboxylase, coproporphyrinogen oxidase, protoporphyrinogen oxidase and ferrochelatase). PEPT1 expression was also negligible in both experimental conditions.

\section{CONCLUSION}

High ALA-induced fluorescence is observed in CFPAC-1 despite significant downregulation of ALA synthase (to reduce endogenous ALA production) and upregulation of ABCB6 (likely due to its ability to export coproporphyrinogen III from the cell). This is probably due to its persistent PEPT1 expression and a lack of significant upregulation of the PpIX exporter ABCG2 despite ALA exposure. In contrast, PANC-1 has negligible PEPT1 expression (the main influx transporter for exogenous ALA in tissues of the digestive tract) and demonstrates significant upregulation of the PpIX exporter ABCG2. H6c7, representing a near-normal genotype to pancreatic ductal epithelial cells, shows significant up- or downregulation of three of the four transporters and six of the eight enzymes involved in the haem biosynthesis pathway, demonstrating a wide-ranging response to exogenous ALA that prevents cellular PpIX accumulation. These mechanisms provide some insight into the reduced PpIX fluorescence seen in non-neoplastic pancreatic tissue compared to some pancreatic cancers and may apply to non-neoplastic tissues in other organs. To improve PpIX-induced fluorescence in ALA-resistant cancers such as PANC-1 may prove challenging. The use of nanocarriers to deliver ALA with ABCG2 inhibitors may improve ALA-induced fluorescence, but may also require inhibition of cell membrane-bound ABCB6. Whilst this may prove technically possible (the molecular weight of cell membrane-bound ABCB6 is different to that of mitochondrial membrane-bound $\mathrm{ABCB} 6$, suggesting morphological differences) ${ }^{8}$; any cross-inhibition would prevent entry of coproporphyrinogen III into the mitochondria leading to a reduction in PpIX and resultant fluorescence. 
Figure 2 Relative mRNA expression of enzymes and transporters involved in the haem biosynthesis pathway in pancreatic cancer cells lines CFPAC-1 and PANC-1 and pancreatic ductal cell line H6c7 with or without 24 hours dark incubation with 0.5mM ALA (expression normalized to HPRT1)
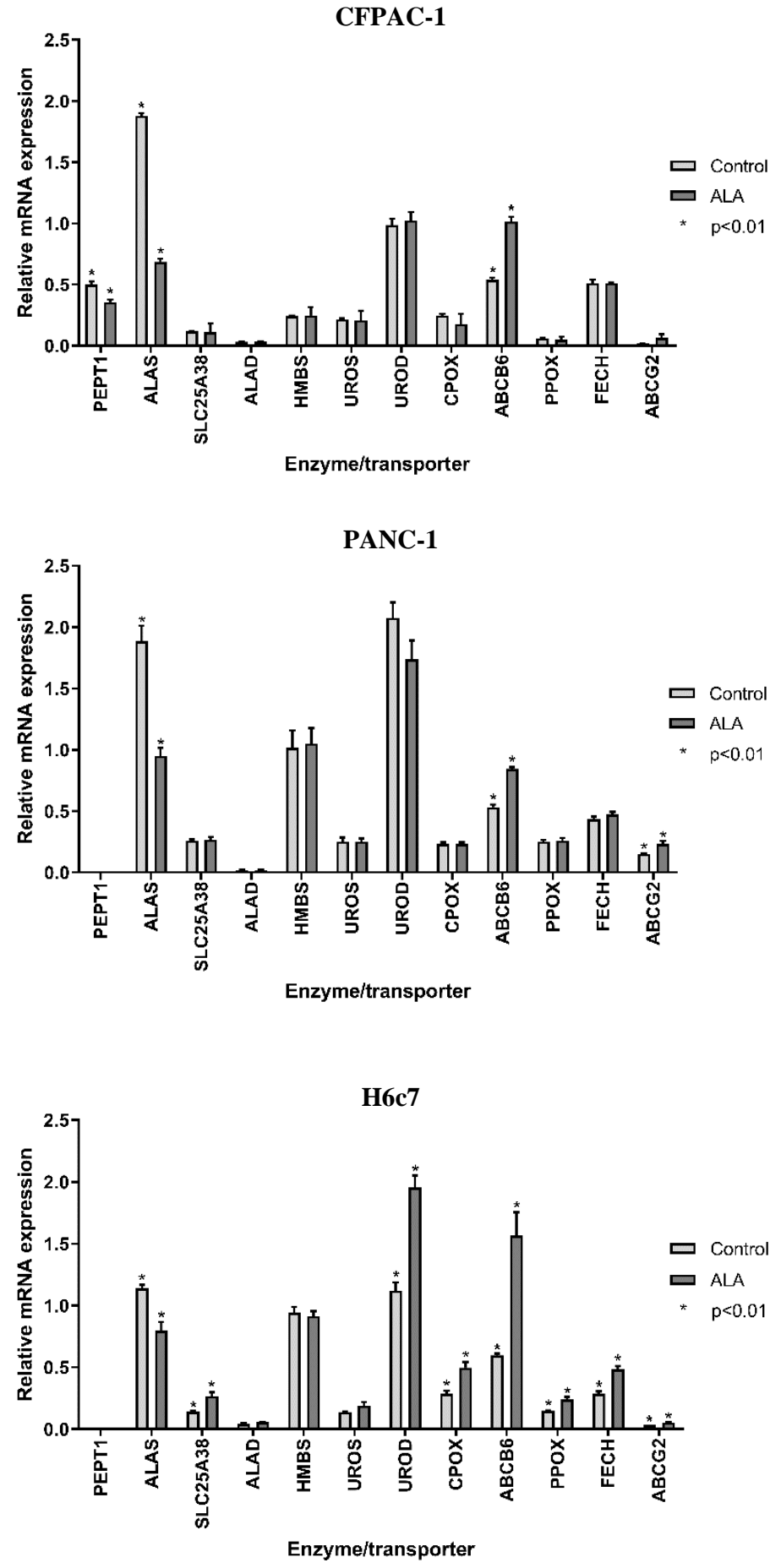

Abbreviations: PEPT1 $=$ Peptide Transporter 1, ALAS $=$ ALA synthase, ALAD = ALA dehydratase, HMBS = hydroxymethylbilane synthase, UROS = uroporphyrinogen III synthase, UROD = uroporphyrinogen decarboxylase, $\mathrm{CPOX}=$ coproporphyrinogen oxidase, $\mathrm{PPOX}=$ protoporphyrinogen oxidase, $\mathrm{FECH}=$ ferrochelatase. 


\section{REFERENCES}

[1] Cancer Research UK., "Pancreatic cancer mortality statistics" (2019).

[2] Pancreatic Cancer UK., "Pancreatic cancer statistics" (2019).

[3] Groot, V. P., Rezaee, N., Wu, W., Cameron, J. L., Fishman, E. K., Hruban, R. H., Weiss, M. J., Zheng, L., Wolfgang, C. L. and He, J., "Patterns, Timing, and Predictors of Recurrence Following Pancreatectomy for Pancreatic Ductal Adenocarcinoma:," Ann. Surg. 267(5), 936-945 (2018).

[4] Hidalgo, M., Cascinu, S., Kleeff, J., Labianca, R., Löhr, J.-M., Neoptolemos, J., Real, F. X., Van Laethem, J.-L. and Heinemann, V., "Addressing the challenges of pancreatic cancer: Future directions for improving outcomes," Pancreatology 15(1), 8-18 (2015).

[5] Webber, J., Kessel, D. and Fromm, D., "Plasma levels of protoporphyrin IX in humans after oral administration of 5-aminolevulinic acid," J. Photochem. Photobiol. B 37, 151-153 (1997).

[6] Juzeniene, A. and Mikolajewska, P., "5-aminolevulinic acid and its derivatives," [Handbook of photomedicine], M. Hamblin and Y. Huang, Eds., CRC press, London (2014).

[7] Peng, Q., Berg, K., Moan, J., Kongshaug, M. and Nesland, J., "5-aminolevulinic acid-based photodynamic therapy: principles and experimental research," Photochem. Photobiol. 65(2), 235-251 (1997).

[8] Paterson, J., Shukla, S., Black, C., Tachiwada, T., Garfield, S., Wincovitch, S., Ernst, D., Agadir, A., Li, X., Ambudkar, S., Szakacs, G., Akiwama, S. and Gottesman, M., "Human ABCB6 localizes to both the outer mitochondrial membrane and the plasma membrane," Biochemistry 46(33), 9443-9452 (2007). 